\title{
Non-minimal Degree-Sequence-Forcing Triples
}

\author{
Michael D. Barrus \\ Department of Mathematics, University of Illinois, \\ Urbana, IL 61801 \\ mbarrus2@math.uiuc.edu \\ Stephen G. Hartke* \\ Department of Mathematics, University of Nebraska, \\ Lincoln, NE 68588-0130 \\ shartke2@math.unl.edu \\ Mohit Kumbhat \\ Department of Mathematics, University of Illinois, \\ Urbana, IL 61801 \\ kumbhat2@math.uiuc.edu
}

November 26, 2008

\begin{abstract}
Given a set $\mathcal{F}$ of graphs, a graph $G$ is $\mathcal{F}$-free if $G$ does not contain any member of $\mathcal{F}$ as an induced subgraph. We say that $\mathcal{F}$ is a degreesequence-forcing set if, for each graph $G$ in the class $\mathcal{C}$ of $\mathcal{F}$-free graphs, every realization of the degree sequence of $G$ is also in $\mathcal{C}$. A degreesequence-forcing set is minimal if no proper subset is degree-sequenceforcing. We characterize the non-minimal degree-sequence-forcing sets $\mathcal{F}$ when $\mathcal{F}$ has size 3 .

Mathematics Subject Classification (2000): 05C75, 05C07

Keywords: degree-sequence-forcing set, forbidden subgraphs, degree

*Research partially supported by a Maude Hammond Fling Faculty Research Fellowship from the University of Nebraska Research Council.
\end{abstract}


sequence characterization, 2 -switch, potentially $P$-graphic, forcibly $P$ graphic

\section{Introduction}

Given a collection $\mathcal{F}$ of graphs, a graph $G$ is said to be $\mathcal{F}$-free if $G$ contains no induced subgraph isomorphic to an element of $\mathcal{F}$. We say that elements of $\mathcal{F}$ are forbidden subgraphs for the class of $\mathcal{F}$-free graphs. Forbidden subgraph notions have proven fruitful in graph theory, mainly because hereditary families have forbidden induced subgraph characterizations. For example, Kuratowski's Theorem can be rephrased as a statement of which induced subgraphs are forbidden for planar graphs, and the Strong Perfect Graph Theorem [4] characterizes perfect graphs in terms of their forbidden subgraphs.

The degree sequence $d(G)$ of a graph $G$ is the list $\left(d_{1}, d_{2}, \ldots, d_{n}\right)$ of degrees of the vertices of $G$, written in non-increasing order. We say that a graph class $\mathcal{C}$ has a degree sequence characterization if it is possible to determine whether or not a graph $G$ is in $\mathcal{C}$ based solely on the degree sequence of $G$. Degree sequence characterizations do not exist for most graph classes, but are often useful when they do exist.

In [1], the authors addressed the question of which classes of graphs can be characterized both in terms of a small collection of forbidden subgraphs and in terms of their degree sequences. In particular, they defined a collection $\mathcal{F}$ of graphs to be degree-sequence-forcing if whenever any realization of a graphic sequence $\pi$ is $\mathcal{F}$-free, every other realization of $\pi$ is $\mathcal{F}$-free as well.

There are degree-sequence-forcing sets associated with several well-known classes of graphs. The sets $\left\{K_{2}\right\}$ and $\left\{2 K_{1}\right\}$ are the forbidden sets for the edgeless and the complete graphs, respectively, which have trivial degree sequence characterizations. Furthermore, $\left\{2 K_{2}, C_{4}, P_{4}\right\},\left\{2 K_{2}, C_{4}, C_{5}\right\}$, and $\left\{2 K_{2}, C_{4}\right\}$ are sets of forbidden induced subgraphs for the threshold graphs [5], split graphs [8], and pseudo-split graphs [14], respectively; each of these classes has a degree sequence characterization ([11], [12], [14]).

In [1] the authors characterized the degree-sequence-forcing sets of order at most two. In this paper we define a degree-sequence-forcing set $\mathcal{F}$ to be minimal if no proper subset of $\mathcal{F}$ is degree-sequence-forcing. Theorem 1 characterizes the non-minimal degree-sequence-forcing sets $\mathcal{F}$ when $\mathcal{F}$ has order three. In the theorem we denote disjoint union by + and join by $\vee$. 


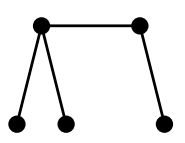

chair

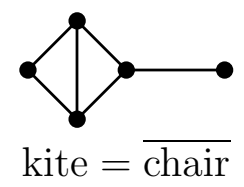

kite $=\overline{\text { chair }}$
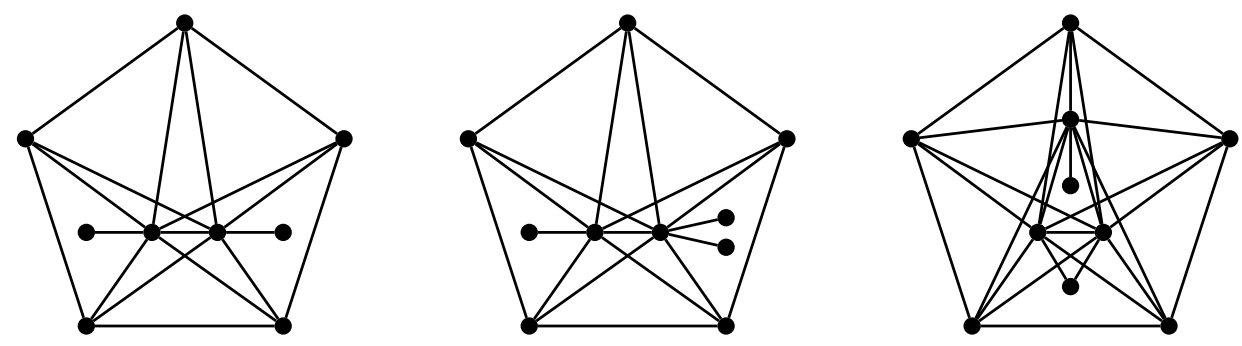

Figure 1: The graphs from Theorem 1(viii).

Theorem 1. A set $\mathcal{F}$ of 3 graphs is a non-minimal degree-sequence-forcing set if and only if one of the following conditions holds:

(1) $\mathcal{F}$ contains a proper degree-sequence-forcing subset other than $\left\{2 K_{2}, C_{4}\right\}$;

(2) $\mathcal{F}=\left\{2 K_{2}, C_{4}, F\right\}$, where $F$ satisfies one of the following:

(i) $F$ induces $2 K_{2}$ or $C_{4}$;

(ii) $F \cong n K_{1}$ or $F \cong K_{n}$ for some $n \geq 1$;

(iii) $F \cong C_{5}+n K_{1}$ or $F \cong C_{5} \vee K_{n}$ for some $n \geq 0$;

(iv) $F \cong\left(\left(C_{5}+n K_{1}\right) \vee K_{1}\right)+m K_{1}$ or $F \cong\left(\left(C_{5} \vee K_{n}\right)+K_{1}\right) \vee K_{m}$ for some $m, n \geq 0$;

(v) $F \cong K_{2}+n K_{1}$ or $F \cong K_{n+2}-e$ for some $n \geq 0$;

(vi) $F$ or $\bar{F}$ is $\left(\left(C_{5} \vee K_{1}\right)+2 K_{1}\right) \vee K_{1}$;

(vii) $F$ has 4 or fewer vertices;

(viii) $F$ is one of the graphs in Figure 1;

(ix) $F \cong K_{1,3}+K_{1}$ or $F \cong\left(K_{3}+K_{1}\right) \vee K_{1}$.

As a consequence of Theorem 1, we place the threshold and split graphs in context by determining all graphs $F$ such that the $\left\{2 K_{2}, C_{4}, F\right\}$-free graphs have a degree sequence characterization.

Our results are particularly interesting in light of recent work done by others in [6], [7], [10], and [15], where small sets $\mathcal{F}$ are characterized that 
force the class of $\mathcal{F}$-free graphs to have a certain property. We find $\mathcal{F}$ for when the property is to have a degree sequence characterization.

The structure of this paper is as follows: Section 2 deals with basic definitions and preliminaries. In Section 3 we show that each of the triples from Theorem 1 is degree-sequence-forcing. In Section 4 we show that there are no other non-minimal degree-sequence-forcing triples by examining an analogue

of degree-sequence-forcing sets in the context of bipartite graphs. Section 5 suggests some directions for future work.

\section{Preliminaries}

In this paper we follow the terminology and notation of West [16]. Specifically, for any graph $G$ and a vertex $v$ of $G$, we use $n(G), N_{G}(v), d_{G}(v)$, and $d(G)=\left(d_{1}, d_{2}, \ldots, d_{n}\right)$ to denote the number of vertices in $G$, the neighborhood of vertex $v$ in $G$, the degree of $v$, and the degree sequence of $G$ in non-increasing order, respectively. We shall denote the disjoint union and join of two graphs $G$ and $H$ by $G+H$ and $G \vee H$, respectively, and the complement of $G$ by $\bar{G}$. We consider all graphs to be simple and to have at least one vertex, particularly when considering induced subgraphs. We will often use "graph" to mean "isomorphism class of graphs," rather than a specific element of a given isomorphism class, unless mentioned otherwise. We trust that the context will make it clear which meaning is intended.

The following theorem from [1] lists all degree-sequence-forcing sets of order at most two.

Theorem 2 ([1]). The degree-sequence-forcing singleton sets $\mathcal{F}$ are $\left\{K_{1}\right\}$, $\left\{K_{2}\right\}$ and $\left\{2 K_{1}\right\}$. The list of all degree-sequence-forcing pairs is as follows:

(i) $\{A, B\}$, where $A$ is one of $K_{1}, K_{2}$, or $2 K_{1}$, and $B$ is arbitrary;

(ii) $\left\{P_{3}, K_{3}\right\},\left\{P_{3}, K_{3}+K_{1}\right\},\left\{P_{3}, K_{3}+K_{2}\right\},\left\{P_{3}, 2 K_{2}\right\},\left\{P_{3}, K_{2}+K_{1}\right\}$;

(iii) $\left\{K_{2}+K_{1}, 3 K_{1}\right\},\left\{K_{2}+K_{1}, K_{1,3}\right\},\left\{K_{2}+K_{1}, K_{2,3}\right\},\left\{K_{2}+K_{1}, C_{4}\right\}$;

(iv) $\left\{K_{3}, 3 K_{1}\right\}$;

(v) $\left\{2 K_{2}, C_{4}\right\}$.

From the definitions given in Section 1, the following two results are immediate: 
Proposition 3 ([1]). Given $\mathcal{F}$ any collection of graphs, let $\mathcal{F}^{c}$ denote the collection of graphs that are complements of those in $\mathcal{F}$. The set $\mathcal{F}$ is degreesequence-forcing if and only if $\mathcal{F}^{c}$ is degree-sequence-forcing.

In particular, $\left\{2 K_{2}, C_{4}, F\right\}$ is degree-sequence-forcing if and only if $\left\{2 K_{2}, C_{4}, \bar{F}\right\}$ is, because $2 K_{2}$ and $C_{4}$ are complements of each other.

A graph $G$ is called a unigraph if it is the only realization of its degree sequence.

Observation 4 ([1]). Given any set $\mathcal{F}$ of graphs, if every $\mathcal{F}$-free graph is a unigraph, then $\mathcal{F}$ is degree-sequence-forcing.

Let $x, y, u, v$ be four vertices of a graph where $x y, u v$ are edges and $x u, y v$ are not edges. A 2-switch is an operation that deletes edges $x y, u v$ and adds $x u, y v$. In this paper, we shall denote such a 2-switch by $\{x y, u v\} \rightrightarrows\{x u, y v\}$. Diagrams showing this 2-switch shall have $x y, u v$ and $x u, y v$ as dotted edges before and after the 2-switch, respectively. Note that for a 2 -switch to be possible the subgraph induced by $x, y, u, v$ must be $2 K_{2}, C_{4}$ or $P_{4}$. Hence any $\left\{2 K_{2}, C_{4}, P_{4}\right\}$-free graph admits no 2 -switch. Also note that any 2 -switch preserves the degree of each vertex of the graph. The following well-known result will be fundamental in what follows.

Theorem 5 (Fulkerson-Hoffman-McAndrew [9]). Graphs $H$ and $H^{\prime}$ on the same vertex set have $d_{H}(v)=d_{H^{\prime}}(v)$ for every vertex $v$ if and only if $H^{\prime}$ can be obtained by performing a sequence of 2-switches on $H$.

We shall call $\left(H, H^{\prime}\right)$ an $\mathcal{F}$-breaking pair if $H$ and $H^{\prime}$ have the same degree sequence, $H$ is not $\mathcal{F}$-free and $H^{\prime}$ is $\mathcal{F}$-free. If $\mathcal{F}=\{F\}$, then we say that the pair $\left(H, H^{\prime}\right)$ is $F$-breaking.

Observation 6. Let $\mathcal{G}$ be a family of graphs. If $\left(H, H^{\prime}\right)$ is a $\left\{2 K_{2}, C_{4}\right\} \cup \mathcal{G}$ breaking pair, then $\left(H, H^{\prime}\right)$ is a $\mathcal{G}$-breaking pair, and $H$ and $H^{\prime}$ are both $\left\{2 K_{2}, C_{4}\right\}$-free.

Proposition 7. If $\mathcal{F}=\left\{2 K_{2}, C_{4}, F\right\}$ is not degree-sequence-forcing, then there exists an $\mathcal{F}$-breaking pair $\left(H, H^{\prime}\right)$ on at most $n(F)+2$ vertices.

Proof. Since $\mathcal{F}$ is not degree-sequence-forcing, by Observation 6 there exists an $\mathcal{F}$-breaking pair $\left(J, J^{\prime}\right)$ of $\left\{2 K_{2}, C_{4}\right\}$-free graphs. By Theorem 5 , there exists some sequence $J=J_{0}, J_{1}, J_{2}, \ldots, J_{k}=J^{\prime}$ of $\left\{2 K_{2}, C_{4}\right\}$-free graphs in which $J_{i}$ is obtained via a 2 -switch on $J_{i-1}$ for $i=1, \ldots, k$. Define $\ell$ to 
be the largest index such that $J_{\ell}$ induces $F$, so that $\left(J_{\ell}, J_{\ell+1}\right)$ is also an $\mathcal{F}$ breaking pair. Let $V$ denote the vertex set of an induced copy of $F$ in $J_{\ell}$, and let $W$ denote the set of 4 vertices involved in the 2-switch transforming $J_{\ell}$ into $J_{\ell+1}$. Since $F$ is not induced on $V$ in $J_{\ell+1}$, the 2 -switch performed must add an edge to or delete an edge from $J_{\ell}[V]$; hence $|W \cap V| \geq 2$ and $|V \cup W| \leq|V|+2$. Thus $\left(J_{\ell}[V \cup W], J_{\ell+1}[V \cup W]\right)$ is an $\mathcal{F}$-breaking pair on at most $n(F)+2$ vertices.

If four vertices $a, b, c, d$ in $G$ induce $P_{4}$ with $a$ and $d$ as the endpoints and $b$ adjacent to $a$, then we denote the path by $\langle a, b, c, d\rangle$ and call $b$ and $c$ the midpoints of the path.

\section{Non-minimal degree-sequence-forcing triples}

In this section we show that each of the triples from Theorem 1 is degreesequence-forcing. Non-minimal triples are formed by appending suitable graphs to the degree-sequence-forcing sets from Theorem 2. The sets that are not $\left\{2 K_{2}, C_{4}\right\}$ are handled easily.

Proposition 8. A set $\mathcal{F}$ with $|\mathcal{F}|=3$ is degree-sequence-forcing if $\mathcal{F}$ contains a degree-sequence-forcing singleton or pair other than $\left\{2 K_{2}, C_{4}\right\}$.

Proof. The authors showed in [1] that any graph that forbids any of the degree-sequence-forcing singletons or pairs other than $\left\{2 K_{2}, C_{4}\right\}$ is a unigraph. Thus any graph that forbids one of these sets and also forbids an additional graph is a unigraph. Observation 4 shows that $\mathcal{F}$ is degree-sequenceforcing.

We now examine the triple $\mathcal{F}=\left\{2 K_{2}, C_{4}, F\right\}$. Not every such triple will be degree-sequence-forcing; for example, the two realizations of $(4,3,3,2,1,1)$ show that $\left\{2 K_{2}, C_{4}, K_{3}+2 K_{1}\right\}$ is not:
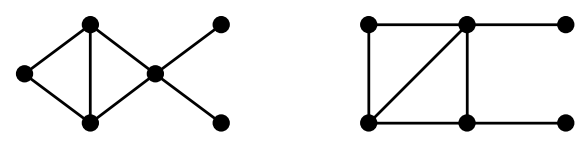

However, there are some triples that are immediately degree-sequence-forcing.

Proposition 9. If $F$ induces either $2 K_{2}$ or $C_{4}$, then $\mathcal{F}$ is degree-sequenceforcing. 
Proof. If $F$ induces $2 K_{2}$ or $C_{4}$, then the $\mathcal{F}$-free graphs are precisely the $\left\{2 K_{2}, C_{4}\right\}$-free graphs, and it follows immediately that $\mathcal{F}$ is degree-sequenceforcing.

We henceforth assume $F$ to be $\left\{2 K_{2}, C_{4}\right\}$-free. Such graphs have been studied in the literature; Blázsik et al. in [3] characterize their structure as follows:

Theorem 10. A graph $G$ is $\left\{2 K_{2}, C_{4}\right\}$-free if and only if there exists a partition $V_{1}, V_{2}, V_{3}$ of $V(G)$ such that (i) $V_{1}$ is an independent set; (ii) $V_{2}$ is a clique; (iii) $V_{3}=\emptyset$ or $G\left[V_{3}\right] \cong C_{5}$; (iv) every possible edge exists between $V_{2}$ and $V_{3}$; and $(v)$ no edge in $G$ has one endpoint in $V_{1}$ and the other endpoint in $V_{3}$.

Given a $\left\{2 K_{2}, C_{4}\right\}$-free graph $G$, we will call the triple $\left(V_{1}, V_{2}, V_{3}\right)$ a pseudo-splitting partition of $V(G)$ if $V_{1}, V_{2}, V_{3}$ satisfy the conditions of the partition set forth in Theorem 10. The name is suggested by [14], in which $\left\{2 K_{2}, C_{4}\right\}$-free graphs are called pseudo-split graphs.

Note that there is at most one induced $C_{5}$ in any $\left\{2 K_{2}, C_{4}\right\}$-free graph. Given a $\left\{2 K_{2}, C_{4}\right\}$-free graph $C$, define the split part $G^{s}$ of $G$ to be the induced subgraph resulting from deleting the vertices of the induced $C_{5}$ from $G$ if such a 5-cycle exists, and letting $G^{s}=G$ otherwise.

In the remainder of this section we present all degree-sequence-forcing triples $\mathcal{F}=\left\{2 K_{2}, C_{4}, F\right\}$ where $F$ is $\left\{2 K_{2}, C_{4}\right\}$-free. We begin with the following easy consequence of Theorem 10.

Observation 11. Let $H$ be an arbitrary $\left\{2 K_{2}, C_{4}\right\}$-free graph, and let $\left(W_{1}, W_{2}, W_{3}\right)$ be a pseudo-splitting partition of $V(H)$. Any induced $P_{4}$ in $H$ either lies in $H\left[W_{3}\right]$ or has its endpoints in $W_{1}$ and its midpoints in $W_{2}$.

Proposition 12. Let $H$ be an arbitrary $\left\{2 K_{2}, C_{4}\right\}$-free graph with pseudosplitting partition $\left(W_{1}, W_{2}, W_{3}\right)$, and let $H^{\prime} ¥ H$ be obtained after performing a 2-switch on $H$. The following statements all hold.

(a) The isomorphism-class-changing 2-switch was performed on a set of vertices in $W_{1} \cup W_{2}$ on which a $P_{4}$ is induced in both $H$ and $H^{\prime}$.

(b) $\left(W_{1}, W_{2}, W_{3}\right)$ is a pseudo-splitting partition of $H^{\prime}$. 
(c) For any $u \in W_{1}, v \in W_{2}$,

$$
\begin{aligned}
& \left|N_{H}(u) \cap W_{2}\right|=\left|N_{H^{\prime}}(u) \cap W_{2}\right| ; \\
& \left|N_{H}(v) \cap W_{1}\right|=\left|N_{H^{\prime}}(v) \cap W_{1}\right| .
\end{aligned}
$$

Proof. (a) The four vertices on which a 2-switch is performed must induce $2 K_{2}, C_{4}$, or $P_{4}$; since the first two graphs are forbidden in $H$, the 2 -switch must have occurred on an induced $P_{4}$. Any 2 -switch on an induced $P_{4}$ leaves an induced $P_{4}$ on the four vertices involved. By Observation 11 the $P_{4}$ must either be located entirely within $W_{3}$ or within $W_{1} \cup W_{2}$. From Theorem 10, any 2-switch on a $P_{4}$ contained in $G\left[W_{3}\right]$ will not change the isomorphism class of the graph, since every 2 -switch on $C_{5}$ produces a $C_{5}$, every vertex of $W_{2}$ dominates the induced $C_{5}$, and every vertex of $W_{1}$ is nonadjacent to every vertex of the induced $C_{5}$. Hence the isomorphism-class-changing 2switch must have occurred on an induced $P_{4}$ in $G\left[W_{1} \cup W_{2}\right]$.

(b) Let $\langle a, b, c, d\rangle$ be the induced $P_{4}$ on which the 2 -switch occurred. By (a) and Observation 11, a, $d \in W_{1}$ and $b, c \in W_{2}$. Note that in the 2-switch the edges deleted are $a b, c d$ and the edges added are $a d, b c$. Thus after the 2 -switch no edge exists between vertices in $W_{1}$, no non-edge exists in $W_{2}$, and all the other requirements for $\left(W_{1}, W_{2}, W_{3}\right)$ to be a pseudo-splitting partition hold.

(c) This is clear upon consideration of the edge deletions and additions under the 2-switch in the proof of (b).

With the following lemma we can present our first examples of degreesequence-forcing $\mathcal{F}$.

Lemma 13. Let $H$ be a $\left\{2 K_{2}, C_{4}\right\}$-free graph with pseudo-splitting partition $\left(W_{1}, W_{2}, W_{3}\right)$, and let $H^{\prime}$ be a graph obtained by performing a 2-switch on $H$. If $F$ is an induced subgraph of $H$ that is not induced in $H^{\prime}$, then $\left|V(F) \cap W_{2}\right| \geq$ 2 .

Proof. Let $H, H^{\prime}$, and $F$ be as described in the hypothesis, and let $V_{2}=$ $V(F) \cap W_{2}$. Since $H\left[W_{1} \cup W_{3}\right] \cong H^{\prime}\left[W_{1} \cup W_{3}\right]$ and $H^{\prime}$ is $F$-free, we have $\left|V_{2}\right| \geq 0$.

Suppose that $\left|V_{2}\right|=1$, and let $V_{2}=\{v\}$. Let $p_{1}$ and $p_{2}$ denote respectively the number of vertices in $V(F) \cap W_{1}$ to which $v$ is and is not adjacent to. By Proposition 12, after the 2-switch that creates $H^{\prime}$ there are still at least $p_{1}$ vertices in $W_{1}$ to which $v$ is adjacent, and at least $p_{2}$ vertices in $W_{1}$ to which 
$v$ is not adjacent. These $p_{1}+p_{2}$ vertices, together with $v$ and $V(F) \cap W_{3}$, clearly induce $F$ in $H^{\prime}$, a contradiction.

Thus $\left|V_{2}\right| \geq 2$, as claimed.

Proposition 14. The set $\mathcal{F}=\left\{2 K_{2}, C_{4}, F\right\}$ is degree-sequence-forcing whenever $F$ is one of the following:

(i) $n K_{1}, n \geq 1$;

(ii) $K_{n}, n \geq 1$;

(iii) $C_{5}+n K_{1}, n \geq 0$;

(iv) $C_{5} \vee K_{n}, n \geq 0$;

(v) $\left(\left(C_{5}+n K_{1}\right) \vee K_{1}\right)+m K_{1}, m, n \geq 0 ;$

(vi) $\left(\left(C_{5} \vee K_{n}\right)+K_{1}\right) \vee K_{m}, m, n \geq 0$.

Proof. If $F$ is any of the graphs described in (i), (iii), or (v), then every pseudo-splitting partition $\left(V_{1}, V_{2}, V_{3}\right)$ of $V(F)$ has $\left|V_{2}\right| \leq 1$. By Lemma 13 and Observation 6 , there exist no $\left\{2 K_{2}, C_{4}, F\right\}$-breaking pairs, so $\left\{2 K_{2}, C_{4}, F\right\}$ is degree-sequence-forcing.

The cases (ii), (iv), and (vi) follow from the cases (i), (iii) and (v) by Proposition 3.

Proposition 15. The triples $\mathcal{F}=\left\{2 K_{2}, C_{4}, K_{2}+n K_{1}\right\}$ and $\mathcal{G}=\left\{2 K_{2}, C_{4}, K_{n+2}-\right.$ e\} are degree-sequence-forcing for all $n \geq 0$.

Proof. By Proposition 3, it suffices to show that the triples $\mathcal{F}$ are degreesequence-forcing.

Proposition 14 handles the case when $n=0$, so assume that $n \geq 1$. Suppose that $\mathcal{F}$ is not degree-sequence-forcing, and let $\left(H, H^{\prime}\right)$ be an $\mathcal{F}$ breaking pair. By Observation $6, H$ and $H^{\prime}$ are a $K_{2}+n K_{1}$-breaking pair where each is $\left\{2 K_{2}, C_{4}\right\}$-free. Let $V=\left\{a, b, i_{1}, \ldots, i_{n}\right\}$ be the vertex set of an induced $K_{2}+n K_{1}$ in $H$, with $a b \in E(H)$. By Proposition 12, we may fix a pseudo-splitting partition $\left(W_{1}, W_{2}, W_{3}\right)$ of both $V(H)$ and $V\left(H^{\prime}\right)$. From Lemma 13 it follows that $\left|V \cap W_{2}\right|=2$, so $V \cap W_{2}=\{a, b\}$, and $V \backslash\{a, b\} \subseteq W_{1}$. Graph $H^{\prime}$ is $K_{2}+n K_{1}$-free, so the 2 -switch transforming $H$ into $H^{\prime}$ must add an edge between either $a$ or $b$ and $i_{k}$ for some $k \in\{1, \ldots, n\}$; without loss of generality assume the edge $a i_{1}$ is added. The 2 -switch must 
be $\left\{a y, x i_{1}\right\} \rightrightarrows\left\{a i_{1}, x y\right\}$ for some $x \in W_{2}$ and some $y \in W_{1}$. However, then $H^{\prime}\left[\left\{a, y, i_{1}, \ldots, i_{n}\right\}\right] \cong K_{2}+n K_{1}$, a contradiction. Thus $\mathcal{F}$ is degree-sequenceforcing.

Proposition 16. The sets $\left\{2 K_{2}, C_{4}, F\right\}$ and $\left\{2 K_{2}, C_{4}, \bar{F}\right\}$ are degree-sequenceforcing, where $F=\left(\left(C_{5} \vee K_{1}\right)+2 K_{1}\right) \vee K_{1}$.

Proof. Suppose $\left(H, H^{\prime}\right)$ is a $\left\{2 K_{2}, C_{4}, F\right\}$-breaking pair, where $F=\left(\left(C_{5} \vee\right.\right.$ $\left.\left.K_{1}\right)+2 K_{1}\right) \vee K_{1}$. By Observation 6 and Proposition 12, we may assume that $H$ induces $F$ and that $H, H^{\prime}$ have the same vertex set and a common pseudo-splitting partition $\left(W_{1}, W_{2}, W_{3}\right)$. Fix a copy of $F$ in $H$. Note that there is a unique pseudo-splitting partition of $F$, and it must be the same as the partition inherited from $H$. Within the induced copy of $F$, let $c$ and $\ell_{1}$ be the vertices of $H$ having degree 8 and 6 , respectively, and let $\ell_{2}$ and $\ell_{3}$ be the pendant vertices. By Proposition 7 , we may assume that $\left(H, H^{\prime}\right)$ is a minimal $F$-breaking pair, in the sense that every vertex in $H$ either belongs to the fixed copy of $F$ or is involved in the 2-switch that creates $H^{\prime}$; then $H$ contains at most 2 vertices not contained in the copy of $F$. In order for there to be an isomorphism-class-changing 2-switch on $H$, there must be an induced $P_{4}$ on $H\left[W_{1} \cup W_{2}\right]$ that includes a vertex from each of $V(F) \cap W_{1}$ and $V(F) \cap W_{2}$. There must then exist some vertex $y$ in $W_{1}$ not contained in the copy of $F, y$ must have some neighbor in $W_{2}$ other than $c$, and $y$ cannot dominate $W_{2}$. If $\left|W_{2}\right|=2$ then $y$ is adjacent to $\ell_{1}$ but not to $c$; but then any 2-switch involving $y$ is of the form $\left\{\ell_{1} y, c v\right\} \rightrightarrows\left\{\ell_{1} v, c y\right\}$ for some $v \in W_{1} \backslash\{y\}$, and $H^{\prime}\left[\{y, w\} \cup W_{2} \cup W_{3}\right]$ is a copy of $F$, where $w \in\left\{\ell_{2}, \ell_{3}\right\}-v$. Thus $H$ contains a vertex $x \in W_{2}$ that does not belong to $F$, and $V(H)=V(F) \cup\{x, y\}$.

If $N(y)=\{x\}$, then the 2-switch changing $H$ into $H^{\prime}$ must be $\{x y, c v\} \rightrightarrows$ $\{x v, c y\}$, where $v \in\left\{\ell_{2}, \ell_{3}\right\}$; since $x v \notin E(H)$, the 2-switch in effect merely switches the roles of $y$ and $v$ without changing the isomorphism class of $H$. A similar contradiction arises if $N(y)=\left\{\ell_{1}\right\}$. The neighborhood of $y$ cannot be $\{x, c\}$ or $\left\{\ell_{1}, c\right\}$ or $\left\{x, c, \ell_{1}\right\}$, for no 2 -switch would then be possible on $H$. Thus $N(y)=\left\{\ell_{1}, x\right\}$, and the 2-switch changing $H$ into $H^{\prime}$ then has the form $\{v y, c w\} \rightrightarrows\{v w, c y\}$, where $v \in\left\{\ell_{1}, x\right\}$ and $w \in\left\{\ell_{2}, \ell_{3}\right\}$. If $v=\ell_{1}$ then $\ell_{1} y \notin E\left(H^{\prime}\right)$, and $H^{\prime}\left[W_{3} \cup\left\{c, \ell_{1}, y, u\right\}\right] \cong F$, where $u \in\left\{\ell_{2}, \ell_{3}\right\}-w$. If $v=x$ then since $x y \notin E\left(H^{\prime}\right)$ and $H^{\prime}$ cannot induce $F$ on $W_{3} \cup\{c, x, y, u\}$, where $u \in\left\{\ell_{2}, \ell_{3}\right\}-w$, we must have $x u \in E\left(H^{\prime}\right)$; but then $H^{\prime}\left[W_{3} \cup\left\{x, \ell_{1}, \ell_{2}, \ell_{3}\right\}\right] \cong$ $F$, a contradiction. 
We conclude that no $\left\{2 K_{2}, C_{4}, F\right\}$-breaking pair exists, so this set is degree-sequence-forcing. By Proposition 3, it follows that $\left\{2 K_{2}, C_{4}, \bar{F}\right\}$ is also degree-sequence-forcing.

Lemma 17. If $G$ is a $\left\{2 K_{2}, C_{4}\right\}$-free graph and $\langle a, b, c, d\rangle$ is an induced $P_{4}$ such that every vertex not in $\{a, b, c, d\}$ is adjacent to exactly 0 or 2 vertices in $\{a, d\}$, then the graph $G^{\prime}$ formed by performing the 2-switch $\{b a, c d\} \rightrightarrows$ $\{b d, c a\}$ is isomorphic to $G$.

Proof. Define $\rho: V(G) \rightarrow V\left(G^{\prime}\right)$ by

$$
\rho(v)= \begin{cases}v, & \text { if } v \notin\{a, d\}, \\ d, & \text { if } v=a, \\ a, & \text { if } v=d .\end{cases}
$$

It is easy to check that $\rho$ is an isomorphism.

Lemma 18. If $P=\langle a, b, c, d\rangle$ is an induced $P_{4}$ in a $\left\{2 K_{2}, C_{4}\right.$, kite $\}$-free graph $G$, then every vertex of $V(G)-\{a, b, c, d\}$ is adjacent to exactly 0 or 2 of $\{a, d\}$.

Proof. Let $\left(V_{1}, V_{2}, V_{3}\right)$ be a pseudo-splitting partition of $G$. By Observation 11, either $P$ is contained within $G\left[V_{3}\right]$, in which case the claim is clearly true by Theorem 10, or $a, d \in V_{1}$ and $b, c \in V_{2}$. Assume the latter holds, and suppose $u \neq b$ and $u a \in E(G)$. Then $u \in V_{2}$, so $u b, u c \in E(G)$. Since $G$ does not induce the kite on $\{a, b, c, d, u\}$, we must have $u d \in E(G)$. Similar arguments show that any vertex other than $c$ adjacent to $d$ must also be adjacent to $a$, and the result follows.

Proposition 19. The $\left\{2 K_{2}, C_{4}\right.$, kite $\}$-free graphs and $\left\{2 K_{2}, C_{4}\right.$, chair $\}$-free graphs are unigraphs.

Proof. Any 2-switch on a $\left\{2 K_{2}, C_{4}\right.$, kite $\}$-free graph $G$ must be performed on an induced $P_{4}$. Lemma 17 and Lemma 18 imply that the graph resulting from such a 2-switch is isomorphic to $G$. It follows from Theorem 5 that $G$ is a unigraph. Since any $\left\{2 K_{2}, C_{4}\right.$, chair $\}$-free graph $H$ is the complement of a $\left\{2 K_{2}, C_{4}\right.$, kite $\}$-free graph, $H$ is also a unigraph.

Corollary 20. The triple $\left\{2 K_{2}, C_{4}, F\right\}$ is degree-sequence-forcing if $F$ is the kite or chair graph, or any graph on 4 or fewer vertices. 
Proof. If $F \in\left\{2 K_{2}, C_{4}\right\}$, then by Proposition 9 the triple is degree-sequenceforcing. If $F \in\left\{4 K_{1}, K_{4}\right\}$, then by Proposition 14 the triple is degreesequence-forcing. If $F$ is any other graph on 4 or fewer vertices, or if $F$ is the kite or chair graph, then $F$ is induced in either the chair or the kite graphs. The $\left\{2 K_{2}, C_{4}, F\right\}$-free graphs are then $\left\{2 K_{2}, C_{4}\right.$, chair $\}$-free or $\left\{2 K_{2}, C_{4}\right.$, kite $\}$-free and hence are unigraphs, so by Observation 4 we have that $\left\{2 K_{2}, C_{4}, F\right\}$ is degree-sequence-forcing.

We now establish some more results about triples $\mathcal{F}=\left\{2 K_{2}, C_{4}, F\right\}$ where $F$ induces $C_{5}$.

Proposition 21. Let $F$ and $H$ be $C_{5}$-inducing graphs that are $\left\{2 K_{2}, C_{4}\right\}$ free, and suppose the vertex set of the split part $F^{s}$ of $F$ has a unique partition into a clique and an independent set. Then $H$ is $F$-free if and only if $H^{s}$ is $F^{s}$-free.

Proof. Suppose first that $H^{s}$ induces $F^{s}$, and assume $V\left(F^{s}\right) \subseteq V\left(H^{s}\right)$. Let $\left(W_{1}, W_{2}, W_{3}\right)$ be the pseudo-splitting partition of $H$. Then $\left(W_{1}, W_{2}\right)$ is a partition of $V\left(H^{s}\right)$ into an independent set and a clique, and if $\left(V_{1}, V_{2}\right)$ is a splitting partition of $V\left(F^{s}\right)$, then the uniqueness of the latter partition forces $V_{1} \subseteq W_{1}$ and $V_{2} \subseteq W_{2}$. Now in $H$ there is an induced $C_{5}$ on $V_{3}$ in which every vertex dominates $W_{2}$. Since $F$ is constructed by making each vertex of a copy of $C_{5}$ adjacent to each vertex in $V_{2}$ and not adjacent to any vertex in $V_{1}$, it is clear that $F$ is induced in $H$.

For the converse, suppose that $H$ induces $F$, and assume $V(F) \subseteq V(H)$. Let $\left(W_{1}, W_{2}, W_{3}\right)$ be a pseudo-splitting partition of $H$, and let $\left(V_{1}, V_{2}, V_{3}\right)$ be a pseudo-splitting partition of $F$. Now $H$ induces a single copy of $C_{5}$, as does $F$, so $V_{3}=W_{3}$. Then $V_{1} \cup V_{2} \subseteq W_{1} \cup W_{2}$, and it is clear that $F^{s}$ is induced in $H^{s}$.

Proposition 22. Suppose $F$ is a $\left\{2 K_{2}, C_{4}\right\}$-free graph such that $F$ induces $C_{5}$, and $V\left(F^{s}\right)$ has a unique partition into a clique and an independent set. If $\left\{2 K_{2}, C_{4}, F^{s}\right\}$ is degree-sequence-forcing, then $\left\{2 K_{2}, C_{4}, F\right\}$ is degreesequence-forcing as well.

Proof. Suppose $\mathcal{F}=\left\{2 K_{2}, C_{4}, F\right\}$ is not degree-sequence-forcing, and let $\left(H_{1}, H_{2}\right)$ be an $\mathcal{F}$-breaking pair. Proposition 21 then implies that $H_{1}^{s}$ induces $F^{s}$, and $H_{2}^{s}$ is $F^{s}$-free; then $\left(H_{1}^{s}, H_{2}^{s}\right)$ is an $F^{s}$-breaking pair and hence a $\left\{2 K_{2}, C_{4}, F^{s}\right\}$-breaking pair. We conclude that $\left\{2 K_{2}, C_{4}, F^{s}\right\}$ is not degreesequence-forcing. 
Corollary 23. Let $F$ be the unique $C_{5}$-inducing $\left\{2 K_{2}, C_{4}\right\}$-free graph such that $F^{s} \cong$ chair. Let $G$ be the unique $C_{5}$-inducing $\left\{2 K_{2}, C_{4}\right\}$-free graph such that $G^{s} \cong P_{4}$ (note that $G$ is self-complementary). The sets $\left\{2 K_{2}, C_{4}, F\right\}$, $\left\{2 K_{2}, C_{4}, \bar{F}\right\}$, and $\left\{2 K_{2}, C_{4}, G\right\}$ are degree-sequence-forcing.

Note that the graphs $F, \bar{F}$, and $G$ are the $C_{5}$-inducing graphs referred to in part (viii) of Theorem 1 .

Whether or not a given set $\mathcal{F}=\left\{2 K_{2}, C_{4}\right\} \cup \mathcal{G}$ is degree-sequence-forcing may be tested in a systematic way via the following algorithm:

Step 1. Generate a list containing, for every $F$ in $\mathcal{G}$, all potentially $F$ inducing-graphic sequences with up to $n(F)+2$ terms.

Step 2. Retain only those sequences having $\left\{2 K_{2}, C_{4}\right\}$-free realizations.

Step 3. For each sequence $\pi$ that remains, test whether or not all realizations of $\pi$ induce some element of $\mathcal{G}$.

Step 4. If some sequence $\pi$ is found in Step 3 that has a $\mathcal{G}$-free realization, then output $\pi$; there is an $\mathcal{F}$-breaking pair having this degree sequence. If no such $\pi$ is found in Step 3, then conclude that $\mathcal{F}$ is degree-sequenceforcing.

It follows easily from Proposition 7 and the appropriate generalization of Observation 6 that the algorithm always successfully concludes whether or not $\mathcal{F}$ is a degree-sequence-forcing set. We note that polynomial-time algorithms exist for Steps 1 and 2 (see [14]); Step 3 may also be automated. We note that for any $F$ in $\mathcal{G}$, any potentially $F$-inducing-graphic sequence that is unigraphic is forcibly $F$-inducing-graphic, so in order to expedite Step 3 , it may be desirable to insert after Step 2 the procedure

Step $2^{\prime}$. Retain only those sequences that are not unigraphic.

A polynomial-time algorithm is given in [13] for this step as well. Using the procedure above the authors obtained the following result.

Proposition 24. The sets $\left\{2 K_{2}, C_{4}, K_{1,3}+K_{1}\right\}$ and $\left\{2 K_{2}, C_{4},\left(K_{3}+K_{1}\right) \vee\right.$ $\left.K_{1}\right\}$ are degree-sequence-forcing. 


\section{Bipartitioned graphs}

In this section we consider a bipartite version of the notion of a degreesequence-forcing set. We then use our results to show that the only nonminimal degree-sequence-forcing triples are the ones presented in Theorem 1.

Define a bipartitioned graph to be a triple $\left(G, V_{1}, V_{2}\right)$ where $G$ is a bipartite graph with partite sets $V_{1}$ and $V_{2}$. We will write $G\left(V_{1}, V_{2}\right)$ for the bipartitioned graph and refer to $G$ as the underlying graph. We define two bipartitioned graphs $G\left(V_{1}, V_{2}\right)$ and $G^{\prime}\left(V_{1}^{\prime}, V_{2}^{\prime}\right)$ to be isomorphic if there exists a graph isomorphism $\phi: V(G) \rightarrow V\left(G^{\prime}\right)$ such that $\phi\left(V_{1}\right)=V_{1}^{\prime}$ (and hence $\left.\phi\left(V_{2}\right)=V_{2}^{\prime}\right)$.

We define the bicomplement $\overline{G\left(V_{1}, V_{2}\right)}$ of a bipartitioned graph $G\left(V_{1}, V_{2}\right)$ to be the bipartitioned graph $H\left(V_{2}, V_{1}\right)$ such that $E(H)=\left\{u v: u \in V_{2}, v \in\right.$ $\left.V_{1}, u v \notin E(G)\right\}$. Note that in the bicomplement the roles of $V_{1}$ and $V_{2}$ are interchanged.

Given a split graph $G$ and a partition $V_{1}, V_{2}$ of $V(G)$ into an independent set and a clique, respectively, we define the associated bipartitioned graph to be $G^{b}\left(V_{1}, V_{2}\right)$, where $G^{b}$ is formed by deleting all edges with both endpoints in $V_{2}$. Note that an arbitrary split graph may have more than one partition into an independent set and a clique, and hence more than one associated bipartitioned graph. Given a $C_{5}$-inducing pseudo-split graph $H$ and a partition $V_{1}, V_{2}, V_{3}$ of $V(H)$ into an independent set, a clique, and the vertex set of an induced $C_{5}$, respectively, define the associated bipartitioned graph to be $H^{b}\left(V_{1}, V_{2}\right)$, where $H^{b}$ is formed by deleting $V_{3}$ from $H$ and removing all edges with both endpoints in $V_{2}$, i.e., $H^{b}=\left(H^{s}\right)^{b}$. A $C_{5}$-inducing pseudo-split graph $H$ has exactly one bipartitioned graph associated with it.

We say a bipartitioned graph $H\left(W_{1}, W_{2}\right)$ is an induced subgraph of $G\left(V_{1}, V_{2}\right)$ if $W_{i} \subseteq V_{i}, i=1,2$, and $H=G\left[W_{1} \cup W_{2}\right]$. We will often be more interested in isomorphism classes of bipartitioned graphs than with specific graphs themselves; for that reason, we say that $G\left(V_{1}, V_{2}\right)$ is $F\left(X_{1}, X_{2}\right)$-free if there is no induced subgraph of $G\left(V_{1}, V_{2}\right)$ isomorphic to $F\left(X_{1}, X_{2}\right)$, and we say that $G\left(V_{1}, V_{2}\right)$ induces $F\left(X_{1}, X_{2}\right)$ if there exists an induced subgraph of $G\left(V_{1}, V_{2}\right)$ isomorphic to $F\left(X_{1}, X_{2}\right)$.

We define the degree sequence of a bipartitioned graph $G\left(V_{1}, V_{2}\right)$ to be the ordered pair $\left(d ; d^{\prime}\right)$, where $d$ and $d^{\prime}$ are lists of the degrees in $G$ of the vertices in $V_{1}$ and $V_{2}$, respectively, written in nonincreasing order. If $G\left(V_{1}, V_{2}\right)$ has degree sequence $\left(d ; d^{\prime}\right)$, we say that $G\left(V_{1}, V_{2}\right)$ is a realization of $\left(d ; d^{\prime}\right)$. 
Example. The chair graph $G$ is shown on the left below. Its unique associated bipartitioned graph $G^{b}\left(V_{1}, V_{2}\right)$ is shown on the right, with vertices in $V_{1}$ on the bottom row and vertices in $V_{2}$ on the top row. The degree sequence of $G^{b}\left(V_{1}, V_{2}\right)$ is $(1,1,1 ; 2,1)$.
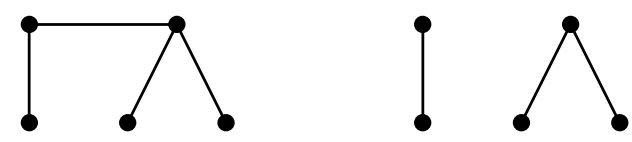

We note that nonisomorphic bipartitioned graphs may have a common degree sequence. We define a set $\mathcal{F}=\left\{F_{1}\left(V_{1}^{1}, V_{2}^{1}\right), \ldots, F_{k}\left(V_{1}^{k}, V_{2}^{k}\right)\right\}$ of bipartitioned graphs to be degree-sequence-forcing if whenever a bipartitioned graph $G\left(W_{1}, W_{2}\right)$ with degree sequence $\left(d ; d^{\prime}\right)$ induces no element of $\mathcal{F}$, then no other realization of $\left(d ; d^{\prime}\right)$ induces an element of $\mathcal{F}$.

In examining degree-sequence-forcing sets of bipartitioned graphs we begin with the following useful note:

Observation 25. Given $\mathcal{F}$ a collection of bipartitioned graphs, let $\mathcal{F}^{c}$ denote the collection of bicomplements of elements of $\mathcal{F}$. Then $\mathcal{F}$ is degree-sequenceforcing if and only if $\mathcal{F}^{c}$ is degree-sequence-forcing.

We define a bipartitioned 2-switch on $G\left(V_{1}, V_{2}\right)$ as the deletion of two edges $u v, x y$ of $G$ and the addition of edges $u y, x v$ not already belonging to $G$, where we require $u, x \in V_{1}$ and $v, y \in V_{2}$, as shown on the left in the diagram below. We again denote this 2 -switch by $\{u v, x y\} \rightrightarrows\{u y, x v\}$. Note that a bipartitioned 2-switch is a 2-switch on the underlying graph. However, the definition a bipartitioned 2-switch is more restrictive; after the 2-switch $\{u v, x y\} \rightrightarrows\{u y, x v\}$ on $G\left(V_{1}, V_{2}\right)$, the sets $V_{1}, V_{2}$ still partition $V(G)$ into two independent sets. Such need not be the case when performing a general 2-switch on a bipartite graph, as shown on the right in the diagram below, where the bottom and top rows of vertices contain subsets of $V_{1}$ and $V_{2}$, respectively.
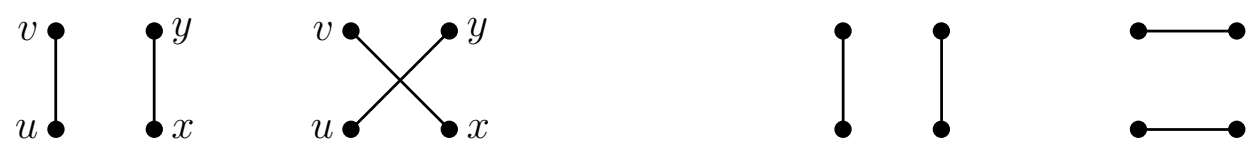

As with general 2-switches, a bipartitioned 2-switch does not change the degree of any vertex in the bipartitioned graph. We noted above that a 
bipartitioned 2-switch does not place any edges between vertices belonging to the same partite set under the given bipartition. We then have the following analogue of Theorem 5:

Proposition 26. Bipartitioned graphs $G\left(W_{1}, W_{2}\right)$ and $H\left(W_{1}, W_{2}\right)$ on the same vertex set satisfy $d_{G}(v)=d_{H}(v)$ for every vertex $v \in W_{1} \cup W_{2}$ if and only if $H$ can be obtained by performing a sequence of bipartitioned 2-switches on $G$.

Proof. We prove this by induction on $\left|W_{1}\right|$. When $\left|W_{1}\right|=1$ there is nothing to prove; consider now bipartitioned graphs $G\left(W_{1}, W_{2}\right)$ and $H\left(W_{1}, W_{2}\right)$ as described in the hypothesis, with $\left|W_{1}\right|>1$. Let $u$ be a vertex of maximum degree $\Delta$ among vertices in $W_{1}$, and let $v_{1}, \ldots, v_{\Delta}$ be a set of vertices in $W_{2}$ with the $\Delta$ highest degrees among vertices in $W_{2}$. We show that by means of bipartitioned 2-switches we can arrive at a bipartitioned graph where $N(u)=\left\{v_{1}, \ldots, v_{\Delta}\right\}$. Suppose $u v_{i} \notin E(G)$ for some $i \in\{1, \ldots, \Delta\}$. Then $u$ has a neighbor $w$ in $W_{2} \backslash\left\{v_{1}, v_{2}, \ldots, v_{\Delta}\right\}$. Since $v_{i}$ has degree at least as large as that of $w, v_{i}$ has a neighbor $x$ in $W_{1} \backslash N(w)$. Then we may perform the 2 -switch $\left\{u w, x v_{i}\right\} \rightrightarrows\left\{u v_{i}, x w\right\}$ and obtain a graph where $\left|N(u) \cap\left\{v_{1}, \ldots, v_{\Delta}\right\}\right|$ is larger than it previously was. Continuing in this way, we may arrive at a graph $G^{*}$ where $N(u)=\left\{v_{1}, \ldots, v_{\Delta}\right\}$. We may also perform a sequence of 2-switches on $H\left(W_{1}, W_{2}\right)$ to form a graph $H^{*}\left(W_{1}, W_{2}\right)$ where $N(u)=\left\{v_{1}, \ldots, v_{\Delta}\right\}$. Now the bipartitioned graphs $G^{*}\left(W_{1}, W_{2}\right)-u$ and $H^{*}\left(W_{1}, W_{2}\right)-u$ agree on the degrees of all vertices, and by the inductive hypothesis there exists a sequence of bipartitioned 2-switches that changes $G^{*}\left(W_{1}, W_{2}\right)-u$ into $H^{*}\left(W_{1}, W_{2}\right)-u$. None of these bipartitioned 2-switches involve the vertex $u$, so the bipartitioned 2-switches that change $G\left(W_{1}, W_{2}\right)$ into $G^{*}\left(W_{1}, W_{2}\right)$, followed by the same bipartitioned 2 -switches that change $G^{*}\left(W_{1}, W_{2}\right)-u$ into $H^{*}\left(W_{1}, W_{2}\right)-u$, followed by the bipartitioned 2-switches that change $H^{*}\left(W_{1}, W_{2}\right)$ into $H\left(W_{1}, W_{2}\right)$, give a sequence of bipartitioned 2-switches that change $G\left(W_{1}, W_{2}\right)$ into $H\left(W_{1}, W_{2}\right)$. The result follows by induction.

We are now in a position to show the relationship between degree-sequenceforcing sets of graphs and degree-sequence-forcing sets of bipartitioned graphs.

Theorem 27. Let $\mathcal{F}$ be a collection of $\left\{2 K_{2}, C_{4}\right\}$-free graphs that either all induce $C_{5}$ or are all $C_{5}$-free. Let $\mathcal{G}$ be the set of all bipartitioned graphs associated with elements of $\mathcal{F}$. Then $\left\{2 K_{2}, C_{4}\right\} \cup \mathcal{F}$ is a degree-sequence-forcing 
set of graphs if and only if $\mathcal{G}$ is a degree-sequence-forcing set of bipartitioned graphs.

Proof. Let $\mathcal{F}$ and $\mathcal{G}$ be as defined above. Suppose first that $\left\{2 K_{2}, C_{4}\right\} \cup \mathcal{F}$ is degree-sequence-forcing. Let $H\left(W_{1}, W_{2}\right)$ be a bipartitioned graph inducing $G\left(V_{1}, V_{2}\right)$, where $G\left(V_{1}, V_{2}\right)$ is an element of $\mathcal{G}$. By definition we have that $V_{1} \subseteq W_{1}$ and $V_{2} \subseteq W_{2}$. Let $H^{\prime}\left(W_{1}, W_{2}\right)$ be any other realization of the degree sequence of $H\left(W_{1}, W_{2}\right)$, and let $J_{1}, J_{2}$ be pseudo-split graphs for which $H$ and $H^{\prime}$ are associated bipartitioned graphs, respectively, where $J_{1}, J_{2}$ induce $C_{5}$ if and only if the graphs in $\mathcal{F}$ do. It is clear that $J_{1}$ and $J_{2}$ have the same degree sequence, and that $J_{1}$ must induce some element of $\mathcal{F}$. Since $\left\{2 K_{2}, C_{4}\right\} \cup \mathcal{F}$ is degree-sequence-forcing, it follows that $J_{2}$ also induces some element of $\mathcal{F}$. Then $H^{\prime}\left(W_{1}, W_{2}\right)$ must induce some element of $\mathcal{G}$. We conclude that $\mathcal{G}$ is degree-sequence-forcing.

Conversely, let $\mathcal{G}$ be degree-sequence-forcing, and suppose that $\left\{2 K_{2}, C_{4}\right\}$ $\cup \mathcal{F}$ is not degree-sequence-forcing. By Observation 6 there exists a $\left\{2 K_{2}, C_{4}\right\}$ $\cup \mathcal{F}$-breaking pair $\left(H_{1}, H_{2}\right)$ of $\left\{2 K_{2}, C_{4}\right\}$-free graphs. Now there exists a sequence of 2-switches that transforms $H_{1}$ into $H_{2}$, and by Proposition 12 there exists a partition $W_{1}, W_{2}, W_{3}$ of $V\left(H_{1}\right)=V\left(H_{2}\right)$ such that in both $H_{1}$ and $H_{2}, W_{1}$ is an independent set, $W_{2}$ is a clique, and $W_{3}$ is either empty or the vertex set of an induced $C_{5}$. Consider the bipartitioned graphs $H_{1}^{b}\left(W_{1}, W_{2}\right)$ and $H_{2}^{b}\left(W_{1}, W_{2}\right)$ associated with $H_{1}$ and $H_{2}$. We have that $H_{1}^{b}\left(W_{1}, W_{2}\right)$ induces $G\left(V_{1}, V_{2}\right)$, where $G\left(V_{1}, V_{2}\right)$ is some element of $\mathcal{G}$. Since $\mathcal{G}$ is degreesequence-forcing, $H_{2}^{b}\left(W_{1}, W_{2}\right)$ also induces some element $G^{\prime}\left(V_{1}^{\prime}, V_{2}^{\prime}\right)$ of $\mathcal{G}$. Let $F$ be the element of $\mathcal{F}$ having $G^{\prime}\left(V_{1}^{\prime}, V_{2}^{\prime}\right)$ as an associated bipartitioned graph. The only way that $F$ may not be induced in $H_{2}$ is for $H_{2}$ to be $C_{5}$-free while $F$ is not. However, if $F$ induces $C_{5}$, then by assumption every element of $\mathcal{F}$ induces $C_{5}$, which implies that $H_{1}$ and hence $H_{2}$ induce $C_{5}$ as well. Thus $H_{2}$ induces an element of $\mathcal{F}$, a contradiction. We conclude that $\left\{2 K_{2}, C_{4}\right\} \cup \mathcal{F}$ is degree-sequence-forcing.

We now apply the technique of monotone parameters, introduced in [1], to give some necessary conditions on degree-sequence-forcing sets of bipartitioned graphs.

Proposition 28. Any degree-sequence-forcing set $\mathcal{G}$ of bipartitioned graphs must contain an element whose underlying graph is a forest.

Proof. For any graph $H$, let $\rho(H)$ denote the number of cycles in $H$. It is clear that $\rho$ is monotone: if $F$ is an induced subgraph of $H$, then $\rho(F) \leq \rho(H)$. 
Let $\mathcal{G}$ be a set of bipartitioned graphs, and let $G\left(V_{1}, V_{2}\right)$ be an element of $\mathcal{G}$ whose underlying graph $G$ minimizes $\rho$. If $\rho(G)>0$, then let $u v$ be an edge of $G$ on a cycle, with $u \in V_{1}$ and $v \in V_{2}$. For vertices $x, y \notin V_{1} \cup V_{2}$, define $V_{1}^{\prime}=V_{1} \cup\{x\}$ and $V_{2}^{\prime}=V_{2} \cup\{y\}$, and define $H\left(V_{1}^{\prime}, V_{2}^{\prime}\right)$ to be the bipartitioned graph whose edge set consists of all edges of $G$, plus the edge $x y$. Let $H^{\prime}\left(V_{1}^{\prime}, V_{2}^{\prime}\right)$ be the bipartitioned graph resulting from the bipartitioned 2-switch $\{u v, x y\} \rightrightarrows\{u y, x v\}$ on $H\left(V_{1}^{\prime}, V_{2}^{\prime}\right)$. It is easily seen that $\rho\left(H^{\prime}\right)<$ $\rho(G)$. By the monotonicity of $\rho$ and the minimality of $G\left(V_{1}, V_{2}\right)$, we have that $H^{\prime}\left(V_{1}^{\prime}, V_{2}^{\prime}\right)$ is $\mathcal{G}$-free. Since $H\left(V_{1}^{\prime}, V_{2}^{\prime}\right)$ has the same degree sequence as $H^{\prime}\left(V_{1}^{\prime}, V_{2}^{\prime}\right)$ and clearly induces an element of $\mathcal{G}$, we see that $\mathcal{G}$ is not degreesequence-forcing. Thus if $\mathcal{G}$ is degree-sequence-forcing, then some element $G\left(V_{1}, V_{2}\right)$ in $\mathcal{G}$ satisfies $\rho(G)=0$, and the result follows.

Proposition 29. Any degree-sequence-forcing set $\mathcal{G}$ of bipartitioned graphs must contain an element whose underlying graph is of the form $K_{\ell, m}+n K_{2}+$ $p K_{1}$ for $\ell, m, n, p \geq 0$.

Proof. For any bipartitioned graph $H\left(V_{1}, V_{2}\right)$, let $\rho\left(H\left(V_{1}, V_{2}\right)\right)$ denote the smallest number of edges that can be added to $H\left(V_{1}, V_{2}\right)$ so that the resulting underlying graph has the form $K_{\ell, m}+n K_{2}+p K_{1}$ and is still bipartite with partite sets $V_{1}, V_{2}$. It is clear that if $F\left(W_{1}, W_{2}\right)$ is induced in $H\left(V_{1}, V_{2}\right)$, then $\rho\left(F\left(W_{1}, W_{2}\right)\right) \leq \rho\left(H\left(V_{1}, V_{2}\right)\right)$. Now let $\mathcal{G}$ be a set of bipartitioned graphs, and let $G\left(V_{1}, V_{2}\right)$ be an element of $\mathcal{G}$ that minimizes $\rho$. Suppose that $\rho\left(G\left(V_{1}, V_{2}\right)\right)>0$. Then choose $u \in V_{1}, v \in V_{2}$ such that $u v$ belongs to a set of $\rho\left(G\left(V_{1}, V_{2}\right)\right)$ edges, each having an endpoint in each of $V_{1}, V_{2}$, that can be added to $G$ to make it of the form $K_{\ell, m}+n K_{2}+p K_{1}$. Now for vertices $x, y \notin V_{1} \cup V_{2}$, define $V_{1}^{\prime}=V_{1} \cup\{x\}$ and $V_{2}^{\prime}=V_{2} \cup\{y\}$, and define $H\left(V_{1}^{\prime}, V_{2}^{\prime}\right)$ to be the bipartitioned graph whose edge set consists of all edges of $G$, plus the edges $u y, x v$. Let $H^{\prime}\left(V_{1}^{\prime}, V_{2}^{\prime}\right)$ be the bipartitioned graph resulting from the bipartitioned 2-switch $\{x v, u y\} \rightrightarrows\{u v, x y\}$ on $H\left(V_{1}^{\prime}, V_{2}^{\prime}\right)$. It is easily seen that $\rho\left(H^{\prime}\left(V_{1}^{\prime}, V_{2}^{\prime}\right)\right)<\rho\left(G\left(V_{1}, V_{2}\right)\right)$. By the monotonicity of $\rho$ and the minimality of $G\left(V_{1}, V_{2}\right)$, we have that $H^{\prime}\left(V_{1}^{\prime}, V_{2}^{\prime}\right)$ is $\mathcal{G}$-free. Since $H\left(V_{1}^{\prime}, V_{2}^{\prime}\right)$ has the same degree sequence as $H^{\prime}\left(V_{1}^{\prime}, V_{2}^{\prime}\right)$ and clearly induces an element of $\mathcal{G}$, we see that $\mathcal{G}$ is not degree-sequence-forcing. Thus if $\mathcal{G}$ is degree-sequenceforcing, then some element $G\left(V_{1}, V_{2}\right)$ in $\mathcal{G}$ satisfies $\rho\left(G\left(V_{1}, V_{2}\right)\right)=0$, and the result follows.

Corollary 30. Any degree-sequence-forcing set $\mathcal{G}$ of bipartitioned graphs must contain two elements $G\left(V_{1}, V_{2}\right)$ and $H\left(W_{1}, W_{2}\right)$ such that $\overline{G\left(V_{1}, V_{2}\right)}$ has 
a forest for its underlying graph, and $\overline{H\left(W_{1}, W_{2}\right)}$ has an underlying graph of the form $K_{\ell, m}+n K_{2}+p K_{1}$ for some $\ell, m, n, p \geq 0$.

Proof. This follows from Observation 25 and Propositions 28 and 29.

Our first application of these results will be to characterize the degreesequence-forcing singleton sets $\left\{G\left(V_{1}, V_{2}\right)\right\}$ of bipartitioned graphs.

Proposition 31. Bipartitioned graphs $G\left(V_{1}, V_{2}\right)$ and $\overline{G\left(V_{1}, V_{2}\right)}$ both have the property that their underlying graphs are forests and graphs of the form $K_{\ell, m}+$ $n K_{2}+p K_{1}$ where $\ell, m, n, p \geq 0$ if and only if either $\min \left\{\left|V_{1}\right|,\left|V_{2}\right|\right\} \leq 1$ or $G \cong K_{1, m}+K_{n}$, where $1 \leq n \leq 2$.

Proof. It is easy to verify that if $\min \left\{\left|V_{1}\right|,\left|V_{2}\right|\right\} \leq 1$ or $G \cong K_{1, m}+K_{n}$, where $1 \leq n \leq 2$, then $G\left(V_{1}, V_{2}\right)$ and its bicomplement satisfy the properties required. We now prove the converse. Let $G\left(V_{1}, V_{2}\right)$ and its bicomplement have the described properties. Then $G$ is of the form $K_{\ell, m}+n K_{2}+p K_{1}$ for some $\ell, m, n, p \geq 0$ with $\ell \leq m$. Since $G$ is also a forest, we have that $0 \leq \ell \leq 1$.

Suppose first that $\ell=n=0$; then $G \cong(m+p) K_{1}$. Since the bicomplement of $G\left(V_{1}, V_{2}\right)$ is also a forest, either $V_{1}$ or $V_{2}$ contains at most one vertex.

If $\ell=0$ and $n \geq 1$, then $G \cong n K_{2}+(m+p) K_{1}$. Fix an edge $u v$ in $G$. Then for any $x \in V_{1} \backslash\{u, v\}$ and $y \in V_{2} \backslash\{u, v\}$, we must have $x$ adjacent to $y$; otherwise, $u, v, x, y$ belong to a component in $\overline{G\left(V_{1}, V_{2}\right)}$ that is not complete bipartite, a contradiction to our assumption. Thus either $\min \left\{\left|V_{1}\right|,\left|V_{2}\right|\right\}=1$, or $m=p=0$ and $n=2$ and hence $G \cong 2 K_{2} \cong K_{1,1}+K_{2}$.

Suppose instead that $\ell=1$. We may assume that $m \geq 2$ since otherwise we could write $G$ as $n^{\prime} K_{2}+p^{\prime} K_{1}$, which was handled in the previous case. Suppose that $\min \left\{\left|V_{1}\right|,\left|V_{2}\right|\right\} \geq 2$. We may also assume that the component $K_{1, m}$ has its center in $V_{2}$ (otherwise, the bicomplement of $G\left(V_{1}, V_{2}\right)$ contains a star component on 3 or more vertices whose center belongs to $V_{2}$, and we may proceed in the proof with the bicomplement). Then there is some vertex $u$ in $V_{2}$ not belonging to the $K_{1, m}$. If there is another vertex $v$ in $V_{2}$ not belonging to the $K_{1, m}$, then $\overline{G\left(V_{1}, V_{2}\right)}$ is not a forest, so $\left|V_{2}\right|=2$. Since $G$ has the form $K_{1, m}+n K_{2}+p K_{1}, u$ has at most one neighbor in $V_{1}$. Any vertex in $V_{1}$ not contained in the $K_{1, m}$ must be adjacent to $u$; otherwise, $u$ belongs to a component in $\overline{G\left(V_{1}, V_{2}\right)}$ that is not complete bipartite. Hence $G$ is isomorphic to either $K_{1, m}+K_{2}$ or $K_{1, m}+K_{1}$. 
Proposition 32. The set $\mathcal{G}=\left\{G\left(V_{1}, V_{2}\right)\right\}$ is degree-sequence-forcing if and only if $G\left(V_{1}, V_{2}\right)$ satisfies one of the following:

(a) $\min \left\{\left|V_{1}\right|,\left|V_{2}\right|\right\} \leq 1$;

(b) $G \cong 2 K_{2}$;

(c) $G \cong K_{1,2}+K_{n}$, where $1 \leq n \leq 2$.

Proof. Let $\mathcal{G}$ be a degree-sequence-forcing set. By Propositions 28, 29 and 31, and Corollary 30, we have that either $\min \left\{\left|V_{1}\right|,\left|V_{2}\right|\right\} \leq 1$ or $G \cong K_{1, m}+K_{n}$ for $1 \leq n \leq 2$. Suppose first that $G \cong K_{1, m}+K_{2}$, with $\min \left\{\left|V_{1}\right|,\left|V_{2}\right|\right\} \geq 2$. Then $m>0$; suppose that $m \geq 3$. Assume first that the center $y$ of the $K_{1, m}$ in $G$ belongs to $V_{2}$. Let $V_{2}^{\prime}=V_{2} \cup\{x\}$, where $x \notin V_{1} \cup V_{2}$, and form the bipartitioned graph $H_{1}\left(V_{1}, V_{2}^{\prime}\right)$ whose edge set consists of $E(G)$ plus an edge from $x$ to a vertex in $V_{1}$ in each component of $G$. Let $a$ be the neighbor of $x$ belonging to the component of order 2 in $G$, and let $b$ be a leaf of the $K_{1, m}$ to which $x$ is not adjacent in $H_{1}$. Form $H_{2}\left(V_{1}, V_{2}^{\prime}\right)$ by performing on $H_{1}\left(V_{1}, V_{2}^{\prime}\right)$ the bipartitioned 2-switch $\{x a, y b\} \rightrightarrows\{x b, y a\}$. Since $\mathcal{G}$ is degree-sequenceforcing, $H_{2}\left(V_{1}, V_{2}^{\prime}\right)$ induces $G\left(V_{1}, V_{2}\right)$, and to obtain a copy of $G\left(V_{1}, V_{2}\right)$ we must delete $x$, the only vertex of degree 2 in $V_{2}^{\prime}$. However, deleting $x$ yields an isolated vertex in $H_{2}\left(V_{1}, V_{2}^{\prime}\right)$, a contradiction, since $G$ has no isolated vertex. A similar contradiction arises if we assume that $y$ belongs to $V_{1}$; we simply interchange the roles of $V_{1}$ and $V_{2}$. We thus conclude that $m \leq 2$, and hence $G \cong 2 K_{2}$ or $G \cong K_{1,2}+K_{2}$.

Suppose next that $G \cong K_{1, m}+K_{1}$ with $\min \left\{\left|V_{1}\right|,\left|V_{2}\right|\right\} \geq 2$. Then $m>0$ and the isolated vertex $z$ in $G$ belongs to the same partite set as the center $y$ of the $K_{1, m}$. Suppose that $m \geq 3$, and assume that $y$ and $z$ belong to $V_{2}$. Let $V_{2}^{\prime}=V_{2} \cup\{x\}$ and $V_{1}^{\prime}=V_{1} \cup\{a\}$, where $x, a \notin V_{1} \cup V_{2}$, and let $b, c$ be two neighbors of $y$ in $V_{1}$. Form the bipartitioned graph $H_{1}\left(V_{1}^{\prime}, V_{2}^{\prime}\right)$ whose edge set consists of $E(G)$ plus the edges $x c, x a$, and $z a$. Form $H_{2}\left(V_{1}^{\prime}, V_{2}^{\prime}\right)$ by performing on $H_{1}\left(V_{1}^{\prime}, V_{2}^{\prime}\right)$ the bipartitioned 2-switch $\{y b, x a\} \rightrightarrows\{y a, x b\}$. For $H_{2}\left(V_{1}^{\prime}, V_{2}^{\prime}\right)$ to induce $G\left(V_{1}, V_{2}\right), y$ must be the center of the $K_{1, m}$ as it is the only vertex with degree greater than 2 , and $y$ 's neighbors must be the leaves of the copy of $K_{1, m}$. However, both $x$ and $z$ are adjacent to a neighbor of $y$, so $G\left(V_{1}, V_{2}\right)$ is not induced in $H_{2}\left(V_{1}^{\prime}, V_{2}^{\prime}\right)$, a contradiction. A similar argument produces a contradiction when $y$ and $z$ belong to $V_{1}$. We conclude again that $m \leq 2$, which produces the desired result. 
We have shown that the degree-sequence-forcing set $\mathcal{G}$ must satisfy the conditions stated in the proposition. To see that these conditions are sufficient for $\mathcal{G}$ to be degree-sequence-forcing, we apply Theorem 27 to the $C_{5^{-}}$ inducing graphs $F$ from Propositions 14 and 16 and Corollary 23 in Section 3. Every bipartitioned graph of the form described appears as the associated bipartitioned graph of some such $F$.

In preparation for later results we now present a result on the structure of split graphs.

Proposition 33. Suppose $S$ is a split graph with more than one associated bipartitioned graph, up to isomorphism. Then $S$ has exactly two associated bipartitioned graphs $G\left(V_{1}, V_{2}\right)$ and $H\left(W_{1}, W_{2}\right)$, up to isomorphism, with $\left|V_{2}\right|$ being the clique number $\omega(S)$ and $\left|W_{2}\right|=\omega(S)-1$. Furthermore, $G\left(V_{1}, V_{2}\right)$ has some isolated vertex $u \in V_{2}$ and $H\left(W_{1}, W_{2}\right)$ has some vertex $v \in W_{1}$ that dominates $W_{2}$, such that $G\left(V_{1}, V_{2}\right)-u \cong H\left(W_{1}, W_{2}\right)-v$.

Proof. Let $S$ be a split graph. Suppose that $G\left(V_{1}, V_{2}\right)$ and $H\left(W_{1}, W_{2}\right)$ are two bipartitioned graphs associated with $S$, with $\left|V_{2}\right|=\left|W_{2}\right|$. We show that $G\left(V_{1}, V_{2}\right) \cong H\left(W_{1}, W_{2}\right)$. We may assume without loss of generality that $V_{1} \cup V_{2}=W_{1} \cup W_{2}=V(S)$. Note that since $W_{1}$ is an independent set and $V_{2}$ is a clique in $S$, we have $\left|V_{2} \cap W_{2}\right| \geq\left|V_{2}\right|-1$. If $\left|V_{2} \cap W_{2}\right|=\left|V_{2}\right|$, then $V_{2}=W_{2}$ and in fact $G\left(V_{1}, V_{2}\right)=H\left(W_{1}, W_{2}\right)$. Suppose instead that $\left|V_{2} \cap W_{2}\right|=\left|V_{2}\right|-1$. Then we may write $V_{2} \backslash W_{2}=\{v\}$ and $W_{2} \backslash V_{2}=\{w\}$, and we have $v \in W_{1}$ and $w \in V_{1}$. Since $V_{1}$ and $W_{1}$ are independent sets, we find that $N(v) \subseteq W_{2}$ and $N(w) \subseteq V_{2}$. Since $N_{S}(v)$ and $N_{S}(w)$ both contain $V_{2} \cap W_{2}$, it follows that the map $\phi: V(S) \rightarrow V(S)$ given by $\phi(v)=w, \phi(w)=v$, and $\phi(y)=y$ for all $y \notin\{v, w\}$ is an automorphism on $S$ such that $\phi\left(V_{2}\right)=W_{2}$. This same map translates to an isomorphism between bipartitioned graphs; thus we have shown that if $\left|V_{2}\right|=\left|W_{2}\right|$, then $G\left(V_{1}, V_{2}\right) \cong H\left(W_{1}, W_{2}\right)$.

Suppose that $G\left(V_{1}, V_{2}\right)$ and $H\left(W_{1}, W_{2}\right)$ are now two nonisomorphic bipartitioned graphs associated with $S$. Then $\left|V_{2}\right| \neq\left|W_{2}\right|$; assume without loss of generality that $\left|V_{2}\right|>\left|W_{2}\right|$. Since at most one vertex of a maximum clique can belong to $W_{1}$, we have $\omega(S)-1 \leq\left|W_{2}\right|$. Then $\left|V_{2}\right|=\omega(S)$ and $\left|W_{2}\right|=\omega(S)-1$. Let $Q$ be a clique of size $\omega(S)$ in $S$. Since $W_{1}$ is an independent set in $S$, at most one vertex of $Q$ can belong to $W_{1}$; it follows that we may write $Q=W_{2} \cup\{q\}$, where $q$ is some vertex in $W_{1}$. Then $q$ is adjacent to every vertex in $W_{2}$ in $S$ and hence in $H$. Since $W_{1}$ is an independent set, $q$ has no other neighbors in $S$. Then $W_{1} \backslash\{q\}, W_{2} \cup\{q\}$ is a partition of $V(S)$ 
into an independent set and a clique, respectively. Let $H^{*}\left(W_{1} \backslash\{q\}, W_{2} \cup\{q\}\right)$ be the associated bipartitioned graph for this partition. Here the vertex $q$ is an isolated vertex in $W_{2} \cup\{q\}$, and $\left|W_{2} \cup\{q\}\right|=\omega(S)$. As we showed above, $H^{*}\left(W_{1} \backslash\{q\}, W_{2} \cup\{q\}\right)$ is isomorphic to $G\left(V_{1}, V_{2}\right)$. Let $q^{\prime}$ be the image of $q$ under an isomorphism from $H^{*}\left(W_{1} \backslash\{q\}, W_{2} \cup\{q\}\right)$ to $G\left(V_{1}, V_{2}\right)$. Then $q^{\prime}$ is an isolated vertex in $V_{2}$ whose deletion from $G\left(V_{1}, V_{2}\right)$ yields a bipartitioned graph isomorphic to $H\left(W_{1}, W_{2}\right)-q$, as desired.

As a consequence of Proposition 33, if a split graph has two associated bipartitioned graphs, we may express them in the form $G\left(V_{1}, V_{2}+u\right)$ and $H\left(V_{1}+u, V_{2}\right)$ for some graphs $G, H$ on the same vertex set.

Proposition 34. Let $\mathcal{F}=\left\{G\left(V_{1}, V_{2}+u\right), H\left(V_{1}+u, V_{2}\right)\right\}$ be the set of associated bipartitioned graphs of a split graph $S$. Let $G^{\prime}=G-u$ and $H^{\prime}=H-u$. If $\mathcal{F}$ is a degree-sequence-forcing pair then $G^{\prime}\left(V_{1}, V_{2}\right) \cong H^{\prime}\left(V_{1}, V_{2}\right)$ must be one of the following:

(a) $n K_{1}$, with $n \geq 0$ and $\left|V_{2}\right| \leq 1$,

(b) $K_{2}$,

(c) $K_{1,2}$,

(d) $K_{2}+K_{1}$, with $\left|V_{2}\right|=1$,

(e) $K_{1,2}+K_{1}$, with $\left|V_{2}\right|=1$,

(f) bicomplements of the above graphs.

Proof. By Proposition 33 we can consider $\mathcal{F}$ to be of the form $\mathcal{F}=\left\{G\left(V_{1}, V_{2}+\right.\right.$ $\left.u), H\left(V_{1}+u, V_{2}\right)\right\}$ and that $G^{\prime}\left(V_{1}, V_{2}\right) \cong H^{\prime}\left(V_{1}, V_{2}\right)$ as bipartitioned graphs, where $G^{\prime}=G-u$ and $H^{\prime}=H-u$. By Propositions 28 and 29 and Corollary 30 and the fact that the classes of forests, bicomplement of forests, graphs of the form $K_{\ell, m}+n K_{2}+p K_{1}$ and their bicomplements are hereditary under induced subgraphs, we find that $G^{\prime}\left(V_{1}, V_{2}\right) \cong H^{\prime}\left(V_{1}, V_{2}\right)$ must be one of the graphs mentioned in Proposition 31.

Case 1: $\min \left\{\left|V_{1}\right|,\left|V_{2}\right|\right\} \leq 1$.

Subcase 1.1: $\min \left\{\left|V_{1}\right|,\left|V_{2}\right|\right\}=0$. Then all vertices are in one part of the bipartition in both $G^{\prime}$ and $H^{\prime}$, and $\mathcal{F}$ is clearly degree-sequence-forcing.

Subcase 1.2: $\min \left\{\left|V_{1}\right|,\left|V_{2}\right|\right\}=1$. We assume that $\left|V_{2}\right|=1$ since if $\left|V_{1}\right|=1$ then the bicomplement of $G^{\prime}\left(V_{1}, V_{2}\right)$ falls under this case. With $\left|V_{2}\right|=1$, we 
find that $G^{\prime}\left(V_{1}, V_{2}\right) \cong H^{\prime}\left(V_{1}, V_{2}\right) \cong K_{1, m}+n K_{1}$, where $m, n \geq 0$.

Claim 1: If $m \geq 3, n \geq 0$ then $\left\{G\left(V_{1}, V_{2}+u\right), H\left(V_{1}+u, V_{2}\right)\right\}$ is not degreesequence-forcing.

Proof. Graphs $G$ and $H$ are as shown in the figure below. Let $c$ denote the center of the nontrivial star component in $G$, let $v_{1}, \ldots, v_{m}$ denote the leaves adjacent to $c$, and let $a_{1}, \ldots, a_{n}$ denote the isolated vertices in $V_{1}$. Form $H_{1}\left(V_{1}+y, V_{2}+u+x\right)$ by adding to $G$ vertices $x$ and $y$ and edges $x v_{1}, x y, u y$. Let $H_{2}\left(V_{1}+y, V_{2}+u+x\right)$ be the bipartitioned graph resulting from the 2-switch $\left\{c v_{m}, x y\right\} \rightrightarrows\left\{x v_{m}, c y\right\}$. Suppose a copy of $G\left(V_{1}, V_{2}+u\right)$ is induced in $H_{2}\left(V_{1}+y, V_{2}+u+x\right)$. We may obtain this copy by deleting a vertex in each of $V_{1}+y$ and $V_{2}+u+x$. Because of degree and distance conditions, we cannot delete $c$ and must delete $u$ and $x$, a contradiction. We also see that $H$ is not induced in $H_{2}$. Thus $\left\{G\left(V_{1}, V_{2}+u\right), H\left(V_{1}+u, V_{2}\right)\right\}$ is not degree-sequence-forcing.

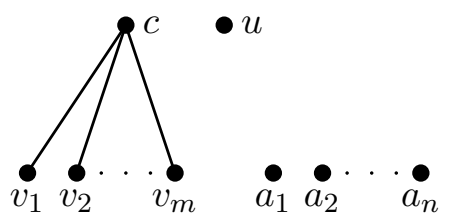

$G$

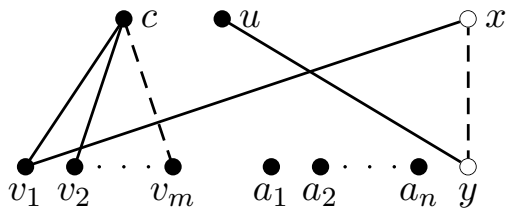

$H_{1}$

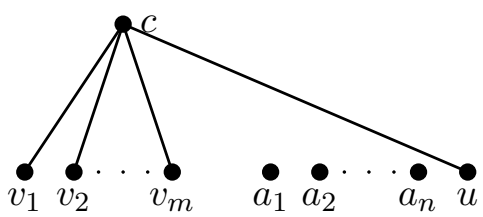

$H$

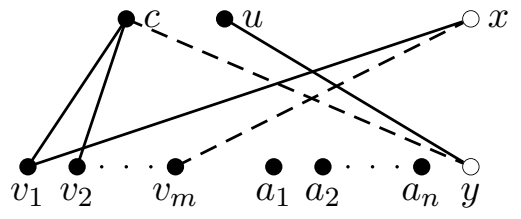

$\mathrm{H}_{2}$

Claim 2: If $m \in\{1,2\}$ and $n \geq 2$, then $\left\{G\left(V_{1}, V_{2}+u\right), H\left(V_{1}+u, V_{2}\right)\right\}$ is not degree-sequence-forcing.

Proof. Graphs $G$ and $H$ are as shown in the figure below (we have illustrated the case $m=2$ ). Again let $c$ denote the center of the nontrivial star component in $G$, let $v_{1}, \ldots, v_{m}$ denote the leaves adjacent to $c$, and let $a_{1}, \ldots, a_{n}$ denote the isolated vertices in $V_{1}$. Form $H_{1}\left(V_{1}+y, V_{2}+u+x\right)$ by adding to $G$ vertices $x$ and $y$ and edges $x y, x a_{1}, x a_{2}, \ldots x a_{n}, u y$. Also add edge $x v_{2}$ if $m=2$. Form bipartitioned graph $H_{2}\left(V_{1}+y, V_{2}+u+x\right)$ by performing on $H_{1}$ the 2 -switch $\left\{c v_{1}, x y\right\} \rightrightarrows\left\{c y, x v_{1}\right\}$. Suppose that $H_{2}\left(V_{1}+y, V_{2}+u+x\right)$ 
induces a copy of $G\left(V_{1}, V_{2}+u\right)$. We may obtain this copy (call it $G^{\prime \prime}\left(W_{1}, W_{2}\right)$ ) by deleting one vertex in $H_{2}$ from each of $V_{1}+y, V_{2}+u+x$. In order to leave $n$ isolated vertices in $W_{1} \cap\left(V_{1}+y\right)$, we must delete $x$; in order to leave an isolated vertex in $W_{2} \cap\left(V_{2}+u+x\right)$, we must delete $y$. However, then no vertex in $W_{2}$ has degree at least $m$ in $G^{\prime \prime}$, a contradiction. Furthermore, if a copy of $H\left(V_{1}+u, V_{2}\right)$ were induced in $H_{2}\left(V_{1}+y, V_{2}+u+x\right)$, then deleting two vertices of $V_{2}+u+x$ would yield this subgraph, and for no pair is this the case. Thus $\left\{G\left(V_{1}, V_{2}+u\right), H\left(V_{1}+u, V_{2}\right)\right\}$ is not degree-sequence-forcing.

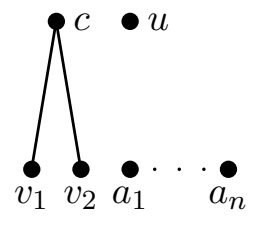

$G$

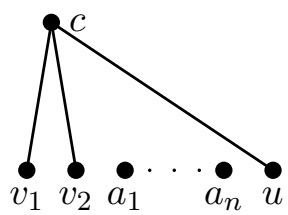

$H$

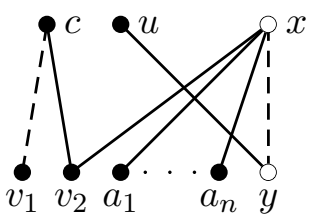

$H_{1}$

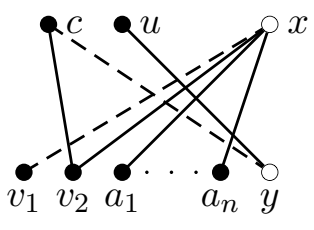

$\mathrm{H}_{2}$

Case 2: $G^{\prime}\left(V_{1}, V_{2}\right) \cong H^{\prime}\left(V_{1}, V_{2}\right) \cong K_{1, m}+K_{n}$ for $1 \leq n \leq 2$. If $m+n \leq 2$ then Case 1 applies, so we assume that $m+n \geq 3$.

Subcase 2.1: $G^{\prime}\left(V_{1}, V_{2}\right) \cong H^{\prime}\left(V_{1}, V_{2}\right) \cong K_{1, m}+K_{1}$, where $m \geq 2$. Then $G$ and $H$ are as shown in the figure below. Form $H_{1}\left(V_{1}+y, V_{2}+u\right)$ by adding to $G$ vertex $y$ and edges $y b, y u$. Let $H_{2}\left(V_{1}+y, V_{2}+u\right)$ be the bipartitioned graph resulting from the 2-switch $\left\{c v_{m}, b y\right\} \rightrightarrows\left\{c y, b v_{m}\right\}$. If a copy of $G\left(V_{1}, V_{2}+u\right)$ is induced in $H_{2}\left(V_{1}+y, V_{2}+u\right)$, it may be obtained by deleting a vertex in $V_{1}+y$. Since $c$ is the only vertex in $V_{2}+u$ having degree $m$, none of its neighbors may be the deleted vertex; however, deleting $v_{m}$ leaves a subgraph not isomorphic to $G$. No vertex of $H_{2}$ has degree $m+1$, so $H\left(V_{1}+u, V_{2}\right)$ is also not an induced subgraph of $H_{2}\left(V_{1}+y, V_{2}+u\right)$. Thus $\left\{G\left(V_{1}, V_{2}+u\right), H\left(V_{1}+u, V_{2}\right)\right\}$ is not degree-sequence-forcing.

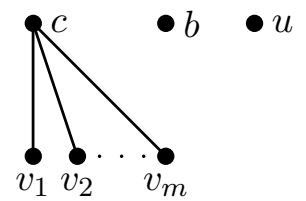

$G$

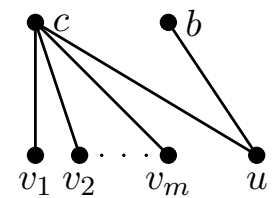

$H$

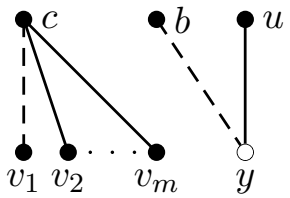

$H_{1}$

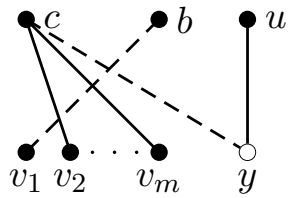

$\mathrm{H}_{2}$

Subcase 2.2: $G^{\prime}\left(V_{1}, V_{2}\right) \cong H^{\prime}\left(V_{1}, V_{2}\right) \cong K_{1, m}+K_{2}$, where $m \geq 1$. Graphs $G$ and $H$ must be as shown in the figure below. Form $H_{1}\left(V_{1}+u+y, V_{2}+x\right)$ by adding to $H$ the vertices $x, y$ and edges $x v$ for $v \in\left\{v_{1}, v_{2} \ldots v_{m}, y\right\}$. Obtain 
$H_{2}\left(V_{1}+u+y, V_{2}+x\right)$ by performing on $H_{1}$ the 2 -switch $\{x y, b u\} \rightrightarrows\{x u, b y\}$. If a copy of $H\left(V_{1}+u, V_{2}\right)$ is induced in $H_{2}\left(V_{1}+u+y, V_{2}+x\right)$, we may isolate it by deleting from $H_{2}$ one vertex in each of $V_{1}+u+y, V_{2}+x$. However, $H$ is connected, and no pair of vertices in $H_{2}$ may be deleted to leave a connected subgraph. Thus $H_{2}\left(V_{1}+u+y, V_{2}+x\right)$ is $H\left(V_{1}+u, V_{2}\right)$-free. Graph $H_{2}\left(V_{1}+u+y, V_{2}+x\right)$ is also $G\left(V_{1}, V_{2}+u\right)$-free, since no two vertices may be deleted to leave in $V_{1}+u+y$ exactly two vertices of degree 1 with different neighbors. Thus $\left\{G\left(V_{1}, V_{2}+u\right), H\left(V_{1}+u, V_{2}\right)\right\}$ is not degree-sequence-forcing.

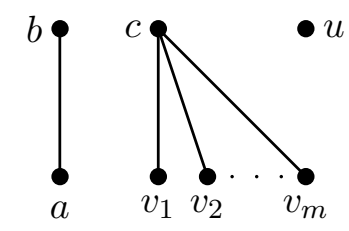

G

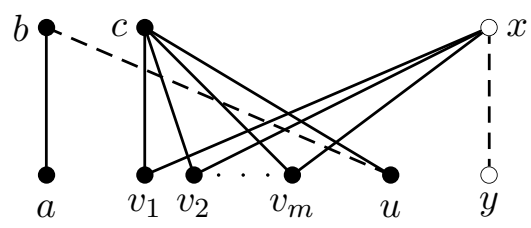

$H_{1}$

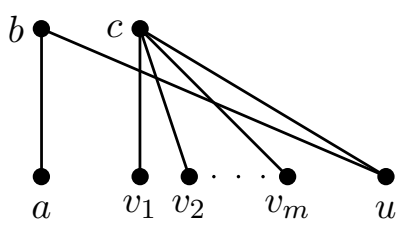

$H$

We conclude with the characterization of all non-minimal degree-sequenceforcing triples of graphs.

Proof of Theorem 1. That the sets listed are degree-sequence-forcing is established in Propositions 8, 9, 14, 15, and 16; Corollaries 20 and 23; and Proposition 24. We now show that these are the only non-minimal degreesequence-forcing triples. We may assume that the triple $\mathcal{F}$ has the form $\left\{2 K_{2}, C_{4}, F\right\}$, where $F$ is $\left\{2 K_{2}, C_{4}\right\}$-free. It follows from Theorem 27 that to characterize $F$ such that $\mathcal{F}$ is degree-sequence-forcing, it suffices to characterize the degree-sequence-forcing sets $\mathcal{G}$ of bipartitioned graphs such that $\mathcal{G}$ consists of the bipartitioned graph or graphs associated with a single pseudosplit graph $F$. Propositions 32 and 34 provide requirements on the structure of $F$, which we now examine in detail. Suppose first that $F$ has a unique 
pseudo-splitting partition; then $F$ induces $C_{5}$, or $F^{s}$ has a unique partition into a clique and independent set. In either case $\mathcal{G}$ consists of a single graph, and from Proposition 32 it follows that $F$ is of a form described in items (iii), (iv), (vi), and (viii) above. Suppose instead that $F$ has more than one pseudo-splitting partition. Then $F$ is split, and $\mathcal{G}$ consists of two graphs as described in Proposition 34. It then follows that $F$ is of the form (ii), (v), (vii), or (ix).

\section{Conclusion}

Theorem 1 provides a complete list of all non-minimal degree-sequenceforcing triples of graphs. Given a triple $\mathcal{F}$ from this list, we have shown that the $\mathcal{F}$-free graphs have a degree sequence characterization, but we have not addressed the question of what the characterization is. Employing the structural characterization of $\left\{2 K_{2}, C_{4}\right\}$-free graphs given in Theorem 10 , the authors have found structural characterizations of the $\mathcal{F}$-free graphs for several such $\mathcal{F}$ 's. These could conceivably lead to simple degree sequence characterizations for these classes, which could in turn lead to linear-time recognition algorithms similar to those known for threshold, split, and pseudo-split graphs. One class in particular that is not well understood at this time is the class of $\left\{2 K_{2}, C_{4}, K_{1,3}+K_{1}\right\}$-free graphs. Finding a structural characterization or degree sequence characterization for this class (or at least a short proof that $\left\{2 K_{2}, C_{4}, K_{1,3}+K_{1}\right\}$ is degree-sequence-forcing that does not rely on a computer) would be interesting.

A complete list of all minimal degree-sequence-forcing triples is not known at present. The authors have shown, as will appear in [2], that for any natural number $k$, there are finitely many minimal degree-sequence-forcing $k$-sets. Thus the problem of listing all minimal degree-sequence-forcing triples is a finite one, and the authors have already identified several of these triples not previously appearing in the literature.

Finally, the characterization of all degree-sequence-forcing triples of the form $\left\{2 K_{2}, C_{4}, F\right\}$ was greatly aided by recasting the degree-sequence-forcing set problem in the context of bipartitioned graphs. In Propositions 32 and

34 we identify all degree-sequence-forcing singleton sets and some pairs of bipartitioned graphs; finding a complete list of degree-sequence-forcing $k$ sets of bipartitioned graphs for any given $k \geq 2$ is an open problem. 


\section{References}

[1] M. D. Barrus, M. Kumbhat, S. G. Hartke, Graph classes characterized both by forbidden subgraphs and degree sequences, J. Graph Theory 57 (2008), no. 2, 131-148.

[2] M. D. Barrus, M. Kumbhat, S. G. Hartke, Minimal degree-sequenceforcing triples, in preparation.

[3] Z. Blázsik, M. Hujter, A. Pluhár, Z. Tuza, Graphs with no induced $C_{4}$ and $2 K_{2}$, Discrete Math. 115 (1993), no. 1-3, 51-55.

[4] M. Chudnovsky, N. Robertson, P. D. Seymour, and R. Thomas, The strong perfect graph theorem, Ann. of Math. (2) 164 (2006), no. 1, 51229 .

[5] V. Chvátal and P. L. Hammer, Aggregation of inequalities in integer programming, In P. L. Hammer, E. L. Johnson, B. H. Korte, and G. L. Nemhauser, editors, Studies in Integer Programming, North-Holland, New York, 1977, 145-162.

[6] R. J. Faudree, R. J. Gould, M. S. Jacobson, and L. L. Lesniak, Characterizing forbidden clawless triples implying Hamiltonian graphs, Discrete Math., 249 (2002), no. 1-3, 71-81.

[7] R. J. Faudree, R. J. Gould, and M. S. Jacobson, Forbidden triples implying Hamiltonicity: for all graphs, Discuss. Math. Graph Theory, 24 (2004), no. 1, 47-54.

[8] S. Földes and P. L. Hammer, On split graphs and some related questions, Problèmes Combinatoires et Théorie Des Graphes, 138-140, Orsay, France, 1976, Colloques internationaux C.N.R.S. 260.

[9] D. R. Fulkerson, A. J. Hoffman, and M. H. McAndrew, Some properties of graphs with multiple edges, Canad. J. Math. 17 (1965), 166-177.

[10] R. J. Gould, T. Łuczak, and F. Pfender, Pancyclity of 3-connected graphs: pairs of forbidden subgraphs, J. Graph Theory, 47 (2004), no. 3, 183-202. 
[11] P. L. Hammer, T. Ibaraki, and B. Simeone, Degree sequences of threshold graphs, Proceedings of the Ninth Southeastern Conference on Combinatorics, Graph Theory, and Computing (Florida Atlantic Univ., Boca Raton, Fla., 1978), 329-355, Congress. Numer., XXI, Utilitas Math., Winnipeg, Man., 1978.

[12] P. L. Hammer and B. Simeone, The splittance of a graph, Combinatorica, 1:275-284, 1981.

[13] D. J. Kleitman and S.-Y. Li, A note on unigraphic sequences, Studies in Appl. Math. 54 (1975), no. 4, 283-287.

[14] F. Maffray and M. Preissmann, Linear recognition of pseduo-split graphs, Disc. Appl. Math., 52 (1994) 307-312.

[15] B. Randerath, 3-colorability and forbidden subgraphs. I. Characterizing pairs, Discrete Math., 276 (2004), no. 1-3, 313-325.

[16] D. B. West, Introduction to graph theory. Prentice Hall, Inc., 1996. 